\title{
Validation of doubled haploid plants by enzymatic mismatch cleavage
}

\author{
Bernhard J Hofinger ${ }^{1}$, Owen A Huynh', Joanna Jankowicz-Cieslak', Andrea Müller², Ingrid Otto², \\ Jochen Kumlehn ${ }^{2}$ and Bradley J Till ${ }^{*}$
}

\begin{abstract}
Background: Doubled haploidy is a fundamental tool in plant breeding as it provides the fastest way to generate populations of meiotic recombinants in a genetically fixed state. A wide range of methods has been developed to produce doubled haploid $(\mathrm{DH})$ plants and recent advances promise efficient $\mathrm{DH}$ production in otherwise recalcitrant species. Since the cellular origin of the plants produced is not always certain, rapid screening techniques are needed to validate that the produced individuals are indeed homozygous and genetically distinct from each other. Ideal methods are easily implemented across species and in crops where whole genome sequence and marker resources are limited.

Results: We have adapted enzymatic mismatch cleavage techniques commonly used for TILLING (Targeting Induced Local Lesions IN Genomes) for the evaluation of heterozygosity in parental, F1 and putative DH plants. We used barley as a model crop and tested 26 amplicons previously developed for TILLING. Experiments were performed using selfextracted single-strand-specific nuclease and standard native agarose gels. Eleven of the twenty-six tested primers allowed unambiguous assignment of heterozygosity in material from F1 crosses and loss of heterozygosity in the DH plants. Through parallel testing of previously developed Simple Sequence Repeat (SSR) markers, we show that 3/32 SSR markers were suitable for screening. This suggests that enzymatic mismatch cleavage approaches can be more efficient than SSR based screening, even in species with well-developed markers.
\end{abstract}

Conclusions: Enzymatic mismatch cleavage has been applied for mutation discovery in many plant species, including those with little or no available genomic DNA sequence information. Here, we show that the same methods provide an efficient system to screen for the production of DH material without the need of specialized equipment. This gene target based approach further allows discovery of novel nucleotide polymorphisms in candidate genes in the parental lines.

Keywords: Polymorphism discovery, TILLING, Single-strand-specific nuclease, Loss of heterozygosity

\section{Background}

The first report of haploid production in plants dates to the early $20^{\text {th }}$ century [1]. The approach is especially powerful in crop breeding because haploid plants are in many cases easily made diploid either spontaneously or via treatment with chemicals such as colchicine. This allows the generation and rapid fixation of genetic variants in a homozygous state. The result is true breeding material with fixed traits that can be used for a variety of approaches in research and crop improvement. The

\footnotetext{
* Correspondence: b.till@iaea.org

'Plant Breeding and Genetics Laboratory, Joint FAO/IAEA Division, International Atomic Energy Agency, Vienna International Centre, PO Box 100, A-1400, Vienna, Austria

Full list of author information is available at the end of the article
}

broad utility of this approach is highlighted by the development of DH methods for more than 250 species [2]. A wide range of techniques have been described for $\mathrm{DH}$ production including pollen embryogenesis, gynogenesis and uniparental genome elimination upon interspecific crosses or pollination by inducer lines [3]. In barley (Hordeum vulgare L.), the so-called bulbosum-technique initially enjoyed a fairly broad application. It relies upon crossings between the two species followed by the spontaneous elimination of the $H$. bulbosum chromosomes, which entails the formation of $H$. vulgare haploids $[4,5]$. Later, however, more efficient and reliable protocols were developed for the production of DHs from immature barley pollen cultivated while either still inside dissected anthers or after isolation [6-8]. These methods

\section{() Biomed Central}


are not only standard in current barley breeding programs, but have also been essential for basic research on pollen embryogenesis $[9,10]$, the development of unique transformation technology and its use in applied research and biotechnology [11-14]. Yet, for all the efforts to produce DHs, efficiencies can vary dramatically for largely unknown reasons and many species and genotypes remain recalcitrant. New approaches may help to address this problem. For example, doubled haploidy could be induced in Arabidopsis thaliana by modification of the centromere-specific histone CENH3 [15]. In theory, this approach could be applied to other species allowing great improvements in plant breeding.

While promising, production of DHs does not occur $100 \%$ of the time. The rate of production can vary depending on species and method used. For example, haploid inducing lines in maize show rates of 8 to $10 \%$ of haploid embryo formation, while all other individuals obtained are unwanted products of regular fertilization events [16]. Moreover, plants can derive from maternal tissue rather than from gametophytic (haploid) cells in anther and ovule/ovary culture [17]. Validation that produced plants are truly haploid or DH is therefore necessary to avoid unwanted heterozygosity in downstream applications. A thorough analysis of putative haploids is especially important when novel methods are developed and when methods are transferred to different laboratories. Without validation, unforeseen decreases in the efficiency of DH production can add years of extra effort to research and breeding projects. We sought to evaluate molecular screening methods to test for homozygosity that are applicable across most crop species. Enzymatic mismatch cleavage methods for polymorphism discovery in heteroduplexed DNA molecules have been widely applied in TILLING reverse-genetics projects $[18,19]$. A standard approach includes PCR amplification of $\sim 1$ to $1.5 \mathrm{~kb}$ gene targets followed by denaturation and annealing to form heteroduplexed molecules that are single stranded where nucleotide polymorphisms exist. This is followed by incubation with a single-strand-specific nuclease that cleaves the DNA at the site of mismatch. The approach is advantageous because with only minor modifications it can be applied to most species. Further, it has proven to be a highly accurate and robust approach for polymorphism discovery in both diploid and polyploid species [20-23]. A range of enzymes has been described for mismatch cleavage including mung bean nuclease, extracts from brassica petioles, and crude extractions from celery $[24,25]$. Double strand breaks can be induced with these enzymes, allowing for low-cost native agarose gels to be used as a readout platform $[26,27]$. When individuals are screened alone using these methods, only heterozygous polymorphisms are detected. If samples are mixed, both homozygous and heterozygous variation can be discovered, allowing unambiguous assignment of polymorphisms. When used to evaluate natural nucleotide polymorphisms, this approach has been termed EcoTILLING [28]. Thus nucleotide diversity and the loss of heterozygosity can be easily evaluated in hundreds of individuals without the need for DNA sequencing [20]. The aim of this study was to adapt these methods for rapid evaluation of loss of heterozygosity in specific gene sequences in putatively $\mathrm{DH}$ plants. Microsatellite marker methods have been previously used to screen for heterozygosity in a non genespecific manner in a variety of plants including barley, Brassica, Mimulus, oil palm and potato [29-33]. We used SSR markers, which are well established in barley, as a baseline to compare the efficiency of enzymatic mismatch cleavage for validating DH material.

\section{Results and discussion}

\section{Development of polymorphic markers for loss of heterozygosity screens}

To evaluate the practical use of an enzymatic mismatch cleavage approach for the screening of homozygosity in putative DH lines of barley, we carried out a pilot experiment to determine the presence of nucleotide polymorphisms in parental plants. We tested the parental lines Golden Promise and HOR1606 and synthetic mixtures of the two parental genomic DNAs with a total of 26 primer pairs that were previously developed for different barley TILLING projects (Table 1). Primer pairs that allow the detection of novel heterozygosity in F1 hybrids not present in the parental lines are considered suitable for DH screening. Five of the tested primer pairs did not amplify a PCR product in at least one of the two parents and were therefore excluded from further experiments (Table 2). The remaining primer pairs produced full-length PCR products in both parents, with four producing substantially lower yields. Consequently, nuclease digestions of these amplicons using a crude celery juice extract (CJE) yielded low concentration cleavage products making visual gel analysis difficult. One primer pair produced a high yield of PCR product, but weak cleavage product banding. Five primer pairs produced a high yield of PCR product but no detectible enzymatic cleavage. The remaining eleven primer pairs produced both high yielding PCR product and high concentration cleaved bands when treated with nuclease, and were thus deemed suitable for DH screening (Figures 1 and 2 and Additional file 1). In addition, cleavage products in synthetic mixtures of genomic DNA of the two parents prior to PCR were observed indicating homozygous polymorphisms between the parents (for example, Figure 1). This resulted in the validation of $11 / 26$ primer pairs suitable for $\mathrm{DH}$ screening.

In order to compare the efficiency of the enzymatic mismatch cleavage approach with an existing method 
Table 1 Primer sequences, PCR product sizes, and references for enzymatic mismatch cleavage

\begin{tabular}{|c|c|c|c|c|}
\hline Number & Primer name & Primer sequence & Product size (bp) & Source \\
\hline \multirow[t]{2}{*}{1} & rdg2a_F1 & CTTGCTCTCAAGACAATGGGTGGATTG & 1499 & 1 \\
\hline & rdg2a_R1 & TCCAAACTGCTAAACATCCGAGGCTCT & & \\
\hline \multirow[t]{2}{*}{2} & rdg2a_F2 & CTCTCAAGACAATGGGTGGATTGCTGA & 1493 & 1 \\
\hline & rdg2a_R2 & CAAACTGCTAAACATCCGAGGCTCTCC & & \\
\hline \multirow[t]{2}{*}{3} & rdg2a_F3 & TCGCTATGTCAAGAGCTGGATGAAGGA & 1496 & 1 \\
\hline & rdg2a_R3 & AAGTGCGTAGGATTGTTCTGCCTTTGC & & \\
\hline \multirow[t]{2}{*}{4} & nbs2-rdg2a_F1 & GCTCTTCCGTITGAAATGAGCAGGAA & 1503 & 1 \\
\hline & nbs2-rdg2a_R1 & TGTITGCATTATGGCCTTGCAAATG & & \\
\hline \multirow[t]{2}{*}{5} & nbs2-rdg2a_F2 & TCCACTACCCGAAAGGCACTCAGCTAC & 1500 & 1 \\
\hline & nb2-rdg2a_R2 & GCAATGCAATGCTCTTACTGACGCAAA & & \\
\hline \multirow[t]{2}{*}{6} & nb2-rdg2a_F3 & TCGAACGAATCAGTGGGTTATGCAAAG & 1497 & 1 \\
\hline & nb2-rdg2a_R3 & ATGAAGTGTTCCCCTCCAGGTTGTCAC & & \\
\hline \multirow[t]{2}{*}{7} & nbs3-rdg2a_F1 & TGGCAAGTCCACTACCAAAAAGGCACT & 1491 & 1 \\
\hline & nbs3-rdg2a_R1 & GCTCTTAGTGATGCCAATACCCGTTGC & & \\
\hline \multirow[t]{2}{*}{8} & nbs3-rdg2a_F2 & GCTCTTCCGTTITGAAATGAGCAGGAA & 1491 & 1 \\
\hline & nbs3-rdg2a_R2 & TGTTTGCATTTATGGCCTTGCAAATG & & \\
\hline \multirow[t]{2}{*}{9} & HVgna1f & GACCCAGATGGCATCCAC & 552 & 2 \\
\hline & HVgna1r & ATGCGACGAGACAAAGGAAT & & \\
\hline \multirow[t]{2}{*}{10} & HVraalf & GTCGACGACTTGCATCATCTATCG & 545 & 2 \\
\hline & HVraa1R & CACCCCGATCACTAACACACAA & & \\
\hline \multirow[t]{2}{*}{11} & HV_hpa1F & СCCTTATGTGTACCCTGATCCTGA & 1100 & 2 \\
\hline & HV_hpa1R & GGTCCAACAGACGTATTAGCCAAG & & \\
\hline \multirow[t]{2}{*}{12} & HV_Mlo9-F1 & AGCAAACCAGACACACAGCAGCGTACC & 900 & 3 \\
\hline & HV_Mlo9-R1 & GCAAAGGCTCACTTTGAGACGGCTTAG & & \\
\hline \multirow[t]{2}{*}{13} & HV_Mlo9-F2 & CATTTGTCGCAAAACAGCAAGTTCGAC & 1476 & 3 \\
\hline & HV_Mlo9-R2 & TTGTCTCATCCCTGGCTGAAGGAAAAA & & \\
\hline \multirow[t]{2}{*}{14} & HvHox1-F1 & AAGCATGGACAAGCATCAGCTCTTTGA & 985 & 3 \\
\hline & HvHox1-R1 & GGCAGCAGCTATCTCGGCTATTITATGG & & \\
\hline \multirow[t]{2}{*}{15} & HV_Kap1_c1_F & TCCACCGGTAAAGAAACCAG & 1030 & 4 \\
\hline & HV_Kap1_C1_R & TGAGGGAGGGAGAAAGATGA & & \\
\hline \multirow[t]{2}{*}{16} & HV_Kap1_d1_F & СTCССТCCСTCAAGAAATCC & 899 & 4 \\
\hline & HV_Kap1_d1_R & GCTGTCGCAAAATACAGCAA & & \\
\hline \multirow[t]{2}{*}{17} & HV_Kap1_e1_F & TTGCTGTATTTTGCGACAGC & 1136 & 4 \\
\hline & HV_Kap1_e1_R & CATGTGTTAAAAGCCGCAGA & & \\
\hline \multirow[t]{2}{*}{18} & HV_Kap1_g1_F & ATTGAGTGCCTCTCGGCTTA & 1175 & 4 \\
\hline & HV_Kap1_g1_R & TGAGGAAAGAAGGGATGTGG & & \\
\hline \multirow[t]{2}{*}{19} & HV_Kap1_h1_F & CСАСАТСССТTСТTССТCA & 718 & 4 \\
\hline & HV_Kap1_h1_R & GGGAGCTTGCCTITCTTCTT & & \\
\hline \multirow[t]{2}{*}{20} & HV_Kap1_i1_F & TGTGGAACTATAAATCTGGCTTCA & 698 & 4 \\
\hline & HV_Kap1_i1_R & CGAGCTAGCCGAACCTGTAG & & \\
\hline \multirow[t]{2}{*}{21} & Hv_mloA-F & CGTGTGCGTACCTGGTAGAG & 599 & 5 \\
\hline & Hv_mloA-R & CAAGCCAAGACGACAATCAG & & \\
\hline \multirow[t]{2}{*}{22} & Hv_mloB-F & CTGATTGTCGTCTTGGCTTG & 624 & 5 \\
\hline & Hv_mloB-R & CTGACTCCATACGCCAAACA & & \\
\hline
\end{tabular}


Table 1 Primer sequences, PCR product sizes, and references for enzymatic mismatch cleavage (Continued)

\begin{tabular}{cllc}
\hline 23 & Hv_mloC-F & TGTTGGCGTATGGAGTCAG & 566 \\
& Av_mloC-R & AGAACCGGAGAGGAGAAGG & 574 \\
& Hv_mloD-F & CCTCACCCTCTTCCTTGACA & 5 \\
& Hv_mloD-R & CGTCAGAGCAGTTCATCAGC & 537 \\
& Hv_mloE-F & CCACCGATGAACTTGTCAGT & 5 \\
& Hv_mloE-R & GAGAGGGGTTTGTTGTGC & 451 \\
& Hv_Mla1-3 F & AGCAGCTCGACAGCCAAGACAA & 5 \\
\hline
\end{tabular}

1J. Jankowicz-Cieslak and B.Till (unpublished).

${ }^{2}[34]$.

${ }^{3}[35]$.

${ }^{4}$ B.Till and F. Taassob Shirazi (unpublished).

${ }^{5}[36]$.

Table 2 Summary of TILLING primers tested, quality of PCR products, CJE digestion products and polymorphisms detected

\begin{tabular}{|c|c|c|c|c|c|c|}
\hline \multirow[t]{2}{*}{ Primer pair } & \multicolumn{2}{|c|}{ PCR product in the parental lines } & \multicolumn{2}{|c|}{ CJE digestion } & \multirow{2}{*}{$\begin{array}{c}\text { Polymorphisms detected } \\
\text { between parental lines }\end{array}$} & \multirow[t]{2}{*}{ Tested in DH material } \\
\hline & $\mathrm{GP}^{1}$ & HOR1606 & $\overline{G P^{2}}$ & HOR 1606 & & \\
\hline rdg2a_F1 + R1 & N & Y & - & - & - & $\mathrm{N}$ \\
\hline rdg2a_F2 + R2 & N & Y & - & - & - & N \\
\hline rdg2a_F3 + R3 & N & W & - & - & - & N \\
\hline nbs2-rdg2a_F1 + R1 & W & W & $?$ & $?$ & N & $\mathrm{N}$ \\
\hline nbs2-rdg2a_F2 + R2 & Y & Y & $\mathrm{F}$ & C & Y & Y \\
\hline nbs2-rdg2a_F3 + R3 & W & W & $?$ & $?$ & N & N \\
\hline nbs3-rdg2a_F1 + R1 & Y & Y & $\mathrm{F}$ & C & Y & Y \\
\hline nbs3-rdg2a_F2 + R2 & N & N & - & - & - & $\mathrm{N}$ \\
\hline$H V g n a 1 f+r$ & N & $N$ & - & - & - & $\mathrm{N}$ \\
\hline HVraalf $+R$ & W & W & $?$ & $?$ & $\mathrm{~N}$ & $\mathrm{~N}$ \\
\hline HV_hpa1F + R & W & W & $?$ & $?$ & $\mathrm{~N}$ & N \\
\hline HV_Mlo9-F1 + R1 & Y & Y & $\mathrm{F}$ & $\mathrm{F}$ & Y & Y \\
\hline HV_Mlo9-F2 + R2 & Y & Y & $\mathrm{F}$ & $\mathrm{F}$ & Y & Y \\
\hline HvHox1-F1 + R1 & Y & Y & $\mathrm{F}$ & $\mathrm{F}$ & N & N \\
\hline HV_Kap1_C1_f +r & Y & Y & $\mathrm{F}$ & $\mathrm{F}$ & Y & Y \\
\hline HV_Kap1_d1_f +r & Y & Y & $\mathrm{F}$ & $\mathrm{F}$ & Y & Y \\
\hline HV_Kap1_e1_f +r & Y & Y & $\mathrm{F}$ & $\mathrm{F}$ & Y & Y \\
\hline HV_Kap1_g1_f +r & Y & Y & $\mathrm{F}$ & $\mathrm{F}$ & N & N \\
\hline HV_Kap1_h1_f + r & Y & Y & $\mathrm{F}$ & $\mathrm{F}$ & N & N \\
\hline HV_Kap1_i1_f + r & Y & Y & $\mathrm{F}$ & $\mathrm{F}$ & N & N \\
\hline$H v \_m l o A-F+R$ & - & - & - & - & Y & Y \\
\hline Hv_mloB-F $+\mathrm{R}$ & - & - & - & - & Y & Y \\
\hline $\mathrm{Hv} \_\mathrm{mloC}-\mathrm{F}+\mathrm{R}$ & - & - & - & - & Y & Y \\
\hline Hv_mloD-F $+\mathrm{R}$ & - & - & - & - & Y & Y \\
\hline $\mathrm{H} v \_\mathrm{mloE}-\mathrm{F}+\mathrm{R}$ & - & - & - & - & $\mathrm{N}$ & N \\
\hline Hv_Mla1-3 F $+3 R$ & - & - & - & - & N & N \\
\hline
\end{tabular}

${ }^{1} \mathrm{Y}=$ yes, $\mathrm{W}=$ weak amplification, $\mathrm{N}=$ no.

${ }^{2} \mathrm{~F}=$ full-length $\mathrm{PCR}$ product, $\mathrm{C}=$ cleavage products present, $?=$ unclear results, $-=$ not tested. 


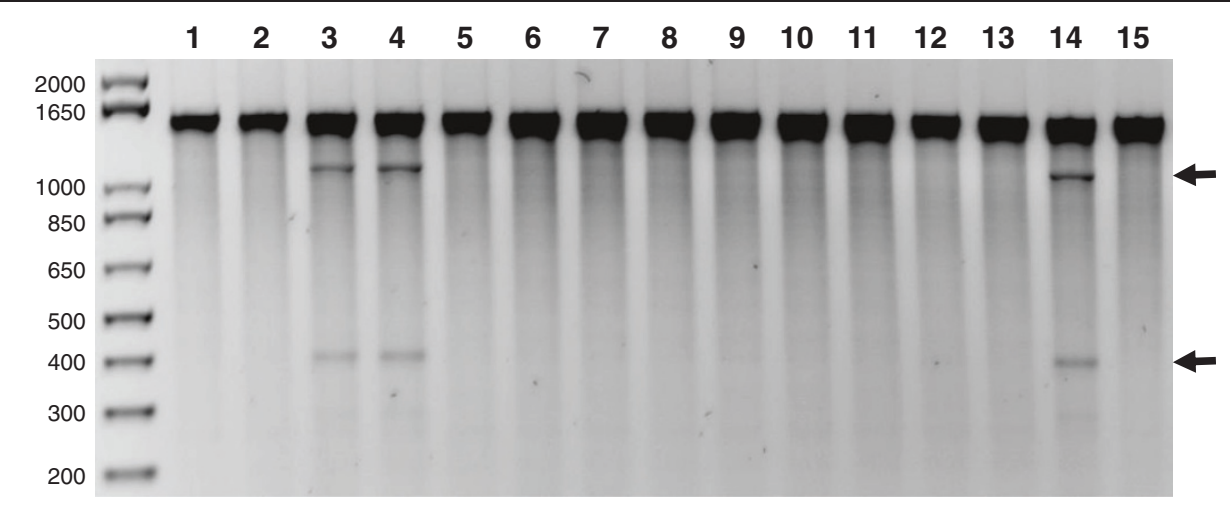

Figure 1 Agarose gel evaluation of doubled haploid production in barley by enzymatic mismatch cleavage. Enzymatic mismatch cleavage was carried out to evaluate homozygosity in putative barley doubled haploid lines. A 1476 bp fragment of the barley Mlo9 gene was PCR amplified and digested with a crude celery juice extract (CJE) containing single-strand-specific nuclease activity followed by agarose gel analysis. The top band in lanes 1-15 represents undigested PCR product. The cleavage products present in heterozygous samples are marked with arrows. Parental lines Golden Promise (GP) and HOR1606 are homozygous for this gene region (lanes 1 and 2 respectively). A synthetic mixture of parental DNA and also the F1 sample from crossing of the two parents show cleavage fragments resulting from a heterozygous SNP (lanes 3 \& 4). Doubled haploid plants (lanes 5-13) are homozygous. Mixtures of genomic DNA from a DH plant and GP show cleavage products while mixture of the same material with HOR1606 does not, indicating the DH harbors the GP allele (lanes 14 \& 15).

based on SSR markers, we tested 32 previously published barley markers. Useful SSR markers are defined as those that amplify a different molecular weight band in each parental material. This allows a clear differentiation between progeny from doubled haploidy versus those maintaining both alleles from the F1 parent. Table 3 summarizes the results of the pilot experiments with the parental lines Golden Promise and HOR1606. Eight primer pairs did not amplify a PCR band in at least one of the two parents. One primer pair could not be used because it amplified several bands. Thirteen primer pairs amplified PCR products in both parents, but no molecular weight polymorphism could be detected between the two parents (Additional file 2). Seven SSR markers did not give a clear result because of limited separation capability of the agarose gels. Although a relatively short length polymorphism cannot be excluded for these markers, they were deemed not suitable for an agarose gel-based screening approach (Additional file 2). Only three of the primer pairs tested showed a clear length polymorphism of the PCR products between Golden Promise and HOR1606 after separation on standard agarose gels, making them candidates for the DH screening experiments.

In summary, the pilot experiments showed that 11 out of 26 (42.3\%) primer pairs are suitable for screening of loss of heterozygosity in DHs using a single-strandspecific nuclease approach. The pilot experiments of the SSR markers revealed only 3 out of 32 (9.4\%) primer pairs were suitable for screening.

\section{Evaluation of homozygosity in DH plants using an enzymatic mismatch cleavage approach}

Based on the pilot experiments, we selected a $1476 \mathrm{bp}$ gene target of the powdery mildew resistance locus

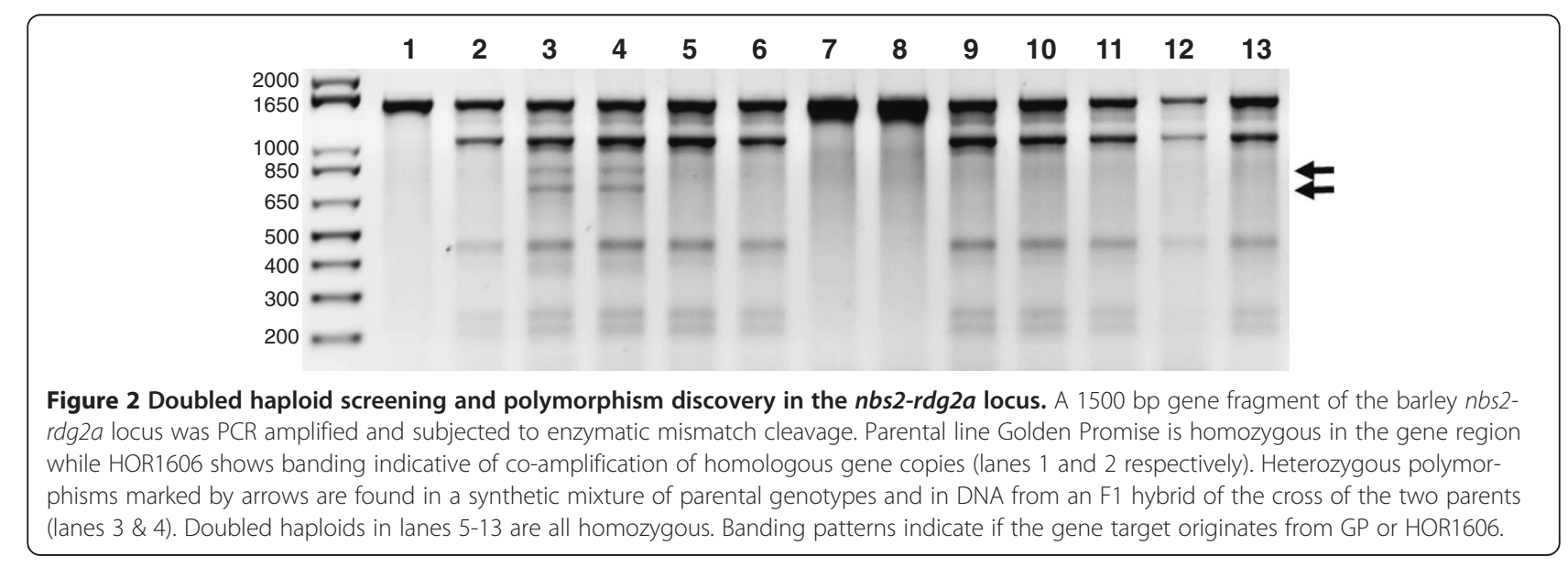


Table 3 Summary of SSR primers tested, quality of PCR products and polymorphisms detected

\begin{tabular}{|c|c|c|c|}
\hline \multirow[t]{2}{*}{ Primer pair $^{1}$} & \multicolumn{2}{|c|}{ PCR amplification $^{2}$} & \multirow{2}{*}{$\begin{array}{c}\text { Polymorphisms detected } \\
\text { between parents }\end{array}$} \\
\hline & GP & HOR1606 & \\
\hline $\mathrm{cnl} 34$ & W & W & $N$ \\
\hline cnl73 & $?$ & $?$ & $N$ \\
\hline $\mathrm{cnl} 31$ & Y & Y & $\mathrm{N}$ \\
\hline cnl146 & Y & Y & Y \\
\hline cnl130 & Y & Y & $?$ \\
\hline cnl151 & $N$ & Y & - \\
\hline cnl140 & $N$ & $N$ & - \\
\hline HVACL1 & Y & Y & $?$ \\
\hline HVLEU & Y & Y & $\mathrm{N}$ \\
\hline HVGNIRE & Y & Y & $N$ \\
\hline HVCSG & $N$ & $N$ & - \\
\hline HVADH1 & $N$ & $N$ & - \\
\hline HWWAXYG & $?$ & $?$ & $N$ \\
\hline HVDHN7 & Y & $?$ & $?$ \\
\hline HVCMA & Y & Y & Y \\
\hline HVDHN9 & Y & Y & $N$ \\
\hline HVBKASI & Y & W & $?$ \\
\hline HVPRP1B & $?$ & Y & $?$ \\
\hline HVBDG & W & W & $N$ \\
\hline HVBARE1 & $N$ & $N$ & - \\
\hline HVRCABG & W & $N$ & - \\
\hline HVSIP1A & Y & Y & $N$ \\
\hline Bmac0113 & Y & Y & Y \\
\hline Bmag0323 & Y & Y & $N$ \\
\hline Bmac0812 & Y & Y & $N$ \\
\hline Bmag0477 & W & W & $?$ \\
\hline Bmag0387 & Y & W & $?$ \\
\hline Bmac0163 & Y & Y & $N$ \\
\hline GBM1284 & $?$ & $?$ & - \\
\hline Bmag0346 & $N$ & $N$ & - \\
\hline Bmag0023 & $N$ & $N$ & - \\
\hline GBM1300 & Y & Y & $N$ \\
\hline
\end{tabular}

${ }^{1}$ From http://germinate.scri.ac.uk/ssr/barley_s.html.

${ }^{2} \mathrm{Y}=$ yes, $\mathrm{W}=$ weak amplification, $\mathrm{N}=$ no, $?=$ unclear results, $-=$ not tested.

Mlo9 to test the applicability of enzymatic mismatch cleavage for evaluation of homozygosity in putative $\mathrm{DH}$ plants of barley [35] (Figure 1). After treatment of the PCR amplification products using CJE, both parental lines, Golden Promise and HOR1606, showed the 1476 bp full-length PCR product indicating homozygosity in the amplified region. In a synthetic mixture of the two parental genomic DNAs before PCR and in F1 plants derived from crossings of the two parents, two cleavage products of approximately $400 \mathrm{bp}$ and $1100 \mathrm{bp}$ were present. These results can be explained by a singlenucleotide polymorphism between the two parents; the molecular weights of the two cleaved fragments sum to the molecular weight of the full-length amplicon. The single-nucleotide polymorphism between the two parental lines was verified by Sanger sequencing (data not shown). All nine DH plants that were produced for this study from the heterozygous F1 generation (see Methods) showed only the $1476 \mathrm{bp}$ full length PCR product. The absence of cleavage bands demonstrated that all of the tested plants are homozygous. To determine the parental origin of the DHs, genomic DNA of the DH material was mixed in equal concentration with DNA from the parental lines prior to PCR and enzymatic digestion. Figure 1 shows an example where mixture with the parental line Golden Promise produced the two mismatch cleavage products. Therefore the genotype of this DH plant in the tested region is different from Golden Promise. As a cross check, a mixture of the same $\mathrm{DH}$ plant with the other parent HOR1606 showed the full-length PCR product but no cleavage products, verifying that this DH plant carries the HOR1606 allele. Similar data was produced with other primer pairs (Additional file 1).

Determination of the parental origin of the DH material also serves as a positive control for enzymatic mismatch cleavage of the heterozygous sites previously determined in F1 material. Each DH individual is assigned to either one or the other (but not both) parental genotypes through the production of cleaved PCR product in the assay. A master mix of CJE is used and applied to all samples and all samples are incubated simultaneously. Thus screening assays contain internal positive and negative controls for enzymatic mismatch cleavage. Previous studies using enzymatic mismatch cleavage for discovery of rare polymorphisms in polyploids and diploids showed false positive and false negative error rates at or below $6 \%$ $[20,37]$. The DH screening procedure presented here is unique to previous work in two important ways. Amplicons are first selected based on discovery of heterozygous polymorphisms. Amplicons where no polymorphisms are detected are not used for screening (Additional file 1). Thus false negative discovery errors are removed before genotyping of the DHs begins. Secondly, the presence of both positive and negative controls in the genotyping assay ensures that enzymatic cleavage activity can be monitored and false positive errors can be detected.

In addition to errors in genotyping, it is also important to consider the chance of misassignment of a plant as being homozygous when it is actually heterozygous. The expected frequencies of not producing a true DH plant will vary depending on the method used for production of DHs and the relative skill of the researcher. The appropriate number of gene targets and samples should 
therefore be screened to control for this. As an example, probabilities can be calculated for assigning a plant as $\mathrm{DH}$ when it is in reality the product of an accidental self-fertilization event. Assuming a normal segregation ratio of 1:2:1, there is a probability of 0.5 that a heterozygous plant is misassigned as homozygous, or $\mathrm{DH}$. When screening 4 unlinked loci, this drops to $0.5^{4}(0.0625)$. This may be a reasonable confidence for many projects. Increasing the number of target loci to 10 gives a $99.9 \%$ chance that the plant is truly homozygous. This should be suitable for even the most valuable material. If segregation bias is a concern, amplicons can be chosen in regions of the genome such as introns where selective pressure is reduced. Indeed, this strategy should result in an increased number of discoverable heterozygous polymorphisms and therefore improve the efficiency of the method. It should be noted that methods for differentiating heterozygous versus homozygous plants are by no means suitable to differentiate haploid versus DH plants. In many crops, this is easily determined phenotypically, e.g. by plant vigour or fertility, while flow cytometry allows for an accurate determination of ploidy even at a very early plant developmental stage.

\section{Evaluation of a more complex target gene for DH screening by enzymatic mismatch cleavage}

In the present study, we discovered two primer pairs that produced a more complex cleavage pattern. Parental line Golden Promise showed the full-length PCR product indicating homozygosity in this gene region, while parent HOR1606 showed cleavage products on the agarose gel in the $n b s 2-r d g 2 a$ and $n b s 3-r d g 2 a$ primer pairs (Figure 2, and Additional file 1B). Sequencing of the PCR product of HOR1606 did not provide any evidence for heterozygosity, but suggested the presence of three very similar gene copies in this genetic background (data not shown). Indeed, positional cloning of the $R d g 2 a$ locus showed three genes whose open reading frames were $87-90 \%$ identical at the DNA level [38]. Thus, the observed complex cleavage pattern is most likely a result of co-amplification of the gene copies during PCR. Cleavage products are observed if nucleotide polymorphisms exist between copies. This phenomenon has been reported in soybean [39]. Further, in polyploid banana, coamplification of homeologous sequences was exploited to evaluate natural nucleotide variation in diverse accessions where the method showed greater than 95\% accuracy in detecting polymorphisms in PCR amplicons [20]. This approach was further exploited to discover EMS induced mutations in TILLING screens [21]. Therefore, the presence of cleavage products in the parental barley line is not expected to adversely affect the ability to screen for loss of heterozygosity. In a synthetic mixture of the two barley parents and in F1 plants derived from crossings between the two parents, all the cleavage bands of HOR1606 were present when screening with primer pair $n b s 2-r d g 2 a$, but also two additional bands of $\sim 800 \mathrm{bp}$ and $700 \mathrm{bp}$ (Figure 2). This additional set of cleavage products indicates at least one single nucleotide polymorphism between the two parental lines. Two of the DH plants examined showed the full-length product like Golden Promise, the other seven DH plants showed the cleavage pattern of HOR1606. This banding pattern indicates the presence of either of the two parental alleles in each DH plant. The absence of the additional bands at $800 \mathrm{bp}$ and $700 \mathrm{bp}$ indicates that all of these plants were homozygous in the region tested. While the banding pattern is more complicated, a cleavage pattern in one of the parents has the advantage that it can be used to track inheritance of the parental genotypes in the DH material without the need of synthetic mixtures with the parental lines (also see Additional file 1B).

Evaluation of homozygosity in DH plants using SSR markers We next examined microsatellite marker Bmac0113 located on chromosome 5 to test DH screening using the SSR approach (Figure 3). The two parental lines Golden Promise and HOR1606 produced each a single PCR band indicating the homozygous state of the marker region. However, there is a clear variation in the size of the two PCR products: approximately $175 \mathrm{bp}$ in Golden Promise and $210 \mathrm{bp}$ in HOR1606. A synthetic mixture of the two parental DNAs and the F1 generation derived from crossings of the two parents showed the presence of both bands at similar intensities indicating heterozygosity. Each of the nine DH plants, which were produced from the F1 generation, showed only a single band verifying the return to a homozygous state in these plants. Six of the DHs inherited the genotype of parent Golden Promise and three of them that of parent HOR1606.

\section{Conclusions}

In this study we developed a low-cost approach utilizing self-extracted enzyme, PCR, and standard agarose gel electrophoresis to evaluate the production of $\mathrm{DHs}$ in barley. Twenty-six primer pairs were needed to identify eleven that were suitable for enzymatic mismatch cleavage screening. For SSR markers, 32 primer pairs were screened in order to identify 3 that were polymorphic in the F1 material. Considering that both methods utilize unlabelled oligonucleotide primers and Taq polymerase, assay costs are roughly comparable. Self-extracted CJE costs less than one cent per reaction. Thus costs are lower in the enzymatic mismatch cleavage approach. The frequency of identifiable heterozygous SNPs in parental material will vary depending on genetic background and target amplicon choice, as will the frequency of suitable SSR primers. Therefore, costs and efficiencies 


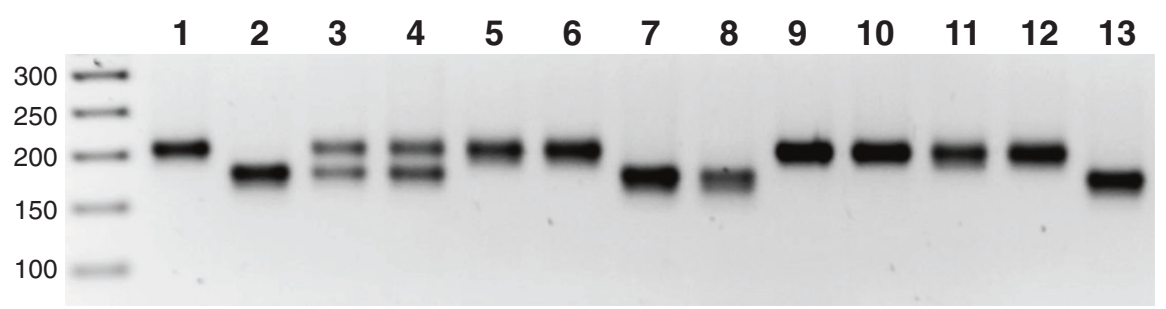

Figure 3 Agarose gel image of evaluation of doubled haploid production in barley using a SSR marker. SSR marker Bmac0113 displays molecular weight polymorphism in amplified bands between parental lines Golden Promise (GP) and HOR1606 (lanes 1 and 2 respectively). Amplification of GP produces a band of approximately $210 \mathrm{bp}$ and HOR1606 produces a band of approximately 175 bp. A synthetic mixture of parental genomic DNA and F1 material from crossing of the two parents shows both bands (lanes 4 \& 5). Doubled haploid plants produce either parental band (lanes 5-13).

of the enzymatic cleavage method are expected to vary. However, a further advantage of enzymatic mismatch cleavage is that the same methods can be applied to most diploid and polyploid species, even where limited DNA sequence information is available. The only requisite is PCR amplification of an appropriately sized amplicon and the presence of polymorphisms between parental materials. Data from this comes from many TILLING projects in different species where only minor modifications of the input genomic DNA concentration for PCR allowed efficient enzymatic mismatch cleavage for the discovery of rare induced mutations [18]. Therefore, we expect enzymatic mismatch cleavage for $\mathrm{DH}$ screening to remain efficient in lesser studied crops that lack well developed marker systems. Further, novel genetic variation between parental lines in specific gene targets of interests can be discovered during the screening process. This gene sequence based approach allows selection of DH plants that are genetically distinct from each other while avoiding unnecessary replication of potentially clonally related material. Additionally, discovery of novel genetic variation in parental lines can provide new insights into gene function. We envision other applications using these methodologies where rapid and low-cost methods for evaluating loss of heterozygosity are sought.

\section{Methods}

\section{Production of DH lines}

Barley cv. 'Golden Promise' that produces light colored grains, and 'Weihenstephaner Schwarze Nackte' (Gatersleben Genbank accession HOR1606) were used as parental lines. For the production of F1 grains, spikes of the female parent 'Golden Promise' were emasculated 2 to 3 days prior to anthesis by removal of the anthers using fine-tipped forceps. Any unintended pollination was prevented by isolating the emasculated spikes in polyethylene bags. When the stigmas were fully receptive, anthers containing mature pollen from the male parent HOR1606 were collected in a plastic petri dish, and then 1 or 2 anthers placed on top of the pistil in each emasculated floret. The bags were kept on the spikes for another week and then removed to avoid fungal contaminations.

F1 hybrids were grown from April to July under fieldlike conditions in partially open, small greenhouses at the campus of IPK Gatersleben. DH lines were produced via pollen embryogenesis. To this end, the spikes were harvested when the most pollen grains were at a stage just prior to mitosis I. Isolation and culture of immature pollen was conducted largely following a method previously described [7]. Specifically, pollen embryogenesis was triggered by spike pretreatment at $4^{\circ} \mathrm{C}$ for three weeks followed by starvation treatment of isolated pollen in SMB medium at $25^{\circ} \mathrm{C}$ for two days, before the pollen was transferred to rich (KBP) medium. To prevent bacterial contamination, the media were supplemented with $100 \mathrm{mg} / \mathrm{L}$ Cefotaxime and $150 \mathrm{mg} / \mathrm{L}$ Timentin.

A total of 10 regenerated plants were established in soil; one was haploid and nine were diploid, as measured by a Ploidy Analyser I (Partec, Münster, Germany) following the manufacturer's instructions. Pure segregation of a single grain color per progeny clearly indicated entire homozygosity of all lines generated. Nine putatively DH lines were used for molecular analysis.

\section{Plant material and growth conditions}

Grains from selfed and crossed parental lines, F1 plants and DH lines as specified above were germinated on wet filter paper and after germination transferred to pots and grown in a greenhouse until the three leaf stage. Barley leaf material was harvested between three to four weeks after planting. One hundred $\mathrm{mg}$ of leaf material was collected in $2 \mathrm{~mL}$ tubes and quick-frozen in liquid nitrogen and either immediately used for DNA isolation or stored at $-80^{\circ} \mathrm{C}$ until DNA isolation.

\section{Isolation of genomic DNA}

Three tungsten carbide beads of $3 \mathrm{~mm}$ diameter (Qiagen, Valencia, CA, USA) were added to $2 \mathrm{~mL}$ tubes containing the leaf samples. Leaves were ground in liquid nitrogen 2 
times using a Qiagen TissueLyser II (10 sec at 1/30 frequency). DNA isolation was carried out with Qiagen DNeasy Plant Mini Kit according to kit instructions or with slightly modified incubation times to increase DNA yield. DNA yield was measured on a NanoDrop 1000 Spectrophotometer (Thermo Scientific, Wilmington, DE, USA) and DNA quality was assessed by gel electrophoresis.

\section{Heteroduplex mismatch cleavage screening}

PCR reactions for enzymatic cleavage were carried out in $25 \mu \mathrm{L}$ volumes under the following conditions (per reaction): 1x Ex $\mathrm{Taq}^{\mathrm{Tm}}$ Reaction Buffer (TaKaRa, Shiga, Japan), $0.2 \mathrm{mM}$ TaKaRa dNTP mixture, $0.5 \mathrm{U}$ TaKaRa Ex Taq ${ }^{\mathrm{TM}}$ Polymerase, $0.12 \mu \mathrm{M}$ of forward and reverse primer and $25 \mathrm{ng}$ genomic DNA. The target genes and sequences of the 26 TILLING primer pairs tested are summarized in Table 1. Design of new primers and PCR amplification was carried out as previously described [19], except that annealing temperatures were adjusted according to the melting temperature of the primer. An annealing temperature of $65^{\circ} \mathrm{C}$ for primer pairs $1-14,55^{\circ} \mathrm{C}$ for primers $15-20$ and $60^{\circ} \mathrm{C}$ for $21-26$ was used (Table 1 ). A $5 \mu \mathrm{L}$ aliquot of the undigested PCR products was separated by agarose gel electrophoresis to check the performance of the PCR. Ten $\mu \mathrm{L}$ aliquots of the PCR products were used for enzymatic mismatch cleavage using a crude CJE containing the nuclease CELI. CJE and reaction buffer were prepared as previously described [19]. Digestion re-

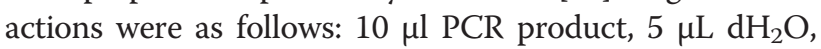
$1.5 \mu \mathrm{L}$ celery juice extract (CJE) buffer and $3.5 \mu \mathrm{L}$ CJE were mixed and incubated for $15 \mathrm{~min}$ at $45^{\circ} \mathrm{C}$. The digestions were stopped by cooling the reaction to $8^{\circ} \mathrm{C}$ and adding $55.56 \mathrm{mM}$ (final concentration) EDTA $(\mathrm{pH}=8.0)$. Ten $\mu \mathrm{L}$ of each reaction were separated by gel electrophoresis on a $1.5 \%$ agarose gel stained with ethidium bromide.

\section{SSR analysis}

Barley SSR primer sequences were taken from Barley SSRs 1.0 (http://germinate.scri.ac.uk/ssr/barley_s.html). PCR reactions for SSR marker amplification were carried out in $25 \mu \mathrm{L}$ volumes under the following conditions (per reaction): $1 \mathrm{x}$ Taq buffer without $\mathrm{MgCl}_{2}$ (Roche Applied Science, Mannheim, Germany), $1.5 \mathrm{mM} \mathrm{MgCl}_{2}$, $0.4 \mathrm{mM}$ PCR nucleotide mix (Promega, Madison, WI, USA), 1.25 U aTaq DNA polymerase (Promega, Madison, WI, USA), $0.32 \mu \mathrm{M}$ of forward and reverse primer and $20 \mathrm{ng}$ genomic DNA. The PCR conditions for SSR marker amplifications were: $5 \mathrm{~min}$ at $94^{\circ} \mathrm{C} ; 35$ cycles $\left(1 \mathrm{~min}\right.$ at $94^{\circ} \mathrm{C} ; 1 \mathrm{~min}$ annealing either at $55^{\circ} \mathrm{C}$ or $58^{\circ} \mathrm{C}$ depending on the SSR marker; $30 \mathrm{sec}$ at $72^{\circ} \mathrm{C}$ ); a final elongation at $72^{\circ} \mathrm{C}$ for $5 \mathrm{~min}$. Aliquots of the PCR products were separated by $2 \%$ agarose gel electrophoresis and visualized by staining with ethidium bromide.

\section{Additional files}

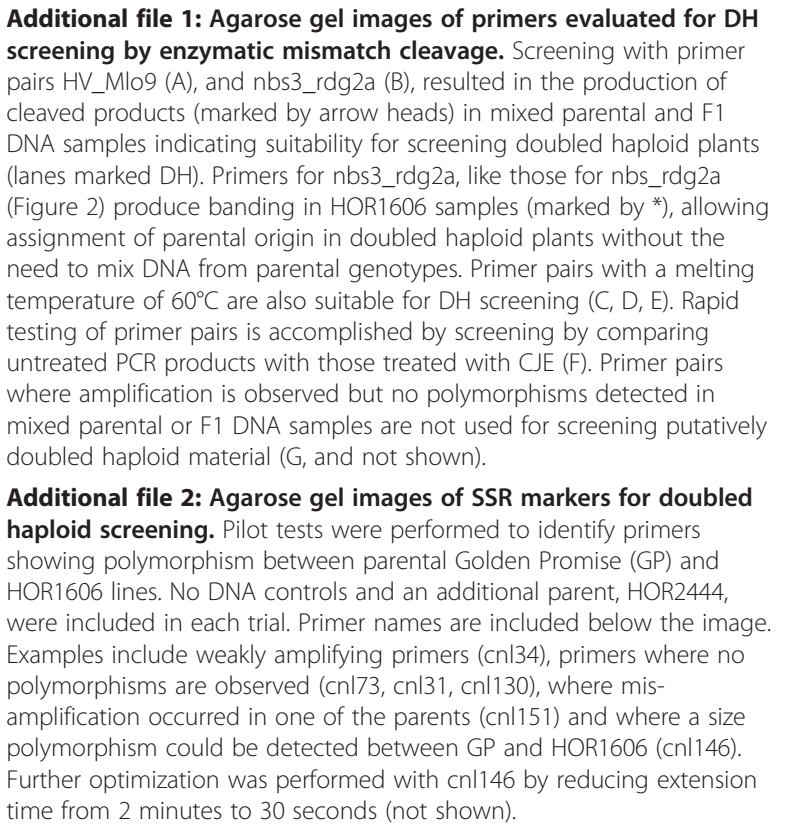

\section{Authors' contributions}

JK and BJT conceived of the study. JK, AM and $1 \mathrm{O}$ participated in the design, execution, data collection and critical analysis of the production of $\mathrm{DH}$ plants. BJH, OAH, JJC and BJT participated in the design, execution, data collection and critical analysis of the enzymatic mismatch cleavage portion of this work. BJH and BJT prepared the manuscript, and JK, OAH and JJC revised it critically. All authors read and approved the final manuscript.

\section{Acknowledgements}

We thank Drs. Iwona Szarejko and Miriam Szurman-Zubrzycka of the University of Silesia, Poland, for sharing their barley TILLING primer sequences. We thank Wahiba Tiliouine of the National Institute for Agricultural Research of Algeria for her assistance in testing primers during a research fellowship with the Plant Breeding and Genetics Laboratory. Farzaneh Taassob Shirazi assisted with Kap1 primer design. Funding for this work was provided by the Food and Agriculture Organization of the United Nations and the International Atomic Energy Agency through their Joint FAO/IAEA Programme of Nuclear Techniques in Food and Agriculture. This work is part of IAEA Coordinated Research Project D24012.

\section{Author details}

${ }^{1}$ Plant Breeding and Genetics Laboratory, Joint FAO/IAEA Division, International Atomic Energy Agency, Vienna International Centre, PO Box 100, A-1400, Vienna, Austria. 'Leibniz Institute of Plant Genetics and Crop Plant Research (IPK) Gatersleben, Plant Reproductive Biology, Corrensstrasse 3, D-06466 Seeland, OT Gatersleben, Germany.

Received: 7 August 2013 Accepted: 1 November 2013 Published: 13 November 2013

\section{References}

1. Blakeslee AF, Belling J, Farnham ME, Bergner AD: A Haploid Mutant in the Jimson Weed, “Datura Stramonium”. Science 1922, 55(1433):646-647.

2. Maluszynski M, Kasha KJ, Szarejko I: Published doubled haploid protocols in plant species. In Doubled Haploid Production in Crop Plants, A Manual. Edited by Maluszynski M, Kasha KJ, Forster BP, Szarejko I. Dordecht: Kluwer Academic Publishers; 2003:309-335.

3. Forster BP, Heberle-Bors E, Kasha KJ, Touraev A: The resurgence of haploids in higher plants. Trends Plant Sci 2007, 12(8):368-375.

4. Kasha K, Kao KN: High frequency haploid production in barley (Hordeum vulgare L.). Nature 1970, 225(5235):874-876. 
5. Pickering RA, Morgan PW: The influence of temperature on chromosome elimination during embryo development in crosses involving Hordeum spp., wheat (Triticum aestivum L.) and rye (Secale cereale L.). Theor Appl Genet 1985, 70:199-206.

6. Hoekstra S, VanZijderveld MH, Louwerse JD, Heidekamp F, Van der Mark F: Anther and microspore culture of Hordeum vulgare L. Cv. Igri. Plant Sci 1992, 86:89-96.

7. Coronado MJ, Hensel G, Broeders S, Otto I, Kumlehn J: Immature pollenderived doubled haploid formation in barley cv. Golden Promise as a tool for transgene recombination. Physiol Plantarum. Acta Physiol Plant 2005, 27:591-599.

8. Oleszczuk S, Sowa S, Zimny J: Androgenic response to preculture stress in microspore cultures of barley. Protoplasma 2006, 228(1-3):95-100.

9. Gonzalez-Melendi P, Ramirez C, Testillano PS, Kumlehn J, Risueno MC: Three dimensional confocal and electron microscopy imaging define the dynamics and mechanisms of diploidisation at early stages of barley microspore-derived embryogenesis. Planta 2005, 222(1):47-57.

10. Daghma DS, Kumlehn J, Hensel G, Rutten T, Melzer M: Time-lapse imaging of the initiation of pollen embryogenesis in barley (Hordeum vulgare L.). $J$ Exp Bot 2012, 63(16):6017-6021.

11. Stein N, Perovic D, Kumlehn J, Pellio B, Stracke S, Streng S, Ordon F, Graner $A$ : The eukaryotic translation initiation factor $4 \mathrm{E}$ confers multiallelic recessive Bymovirus resistance in Hordeum vulgare (L.). Plant J 2005, 42(6):912-922

12. Kumlehn J, Serazetdinova L, Hensel G, Becker D, Loerz H: Genetic transformation of barley (Hordeum vulgare L.) via infection of androgenetic pollen cultures with Agrobacterium tumefaciens. Plant Biotechnol J 2006, 4(2):251-261.

13. Kapusi E, Ma L, Teo CH, Hensel G, Himmelbach A, Schubert I, Mette MF, Kumlehn J, Houben A: Telomere-mediated truncation of barley chromosomes. Chromosoma 2012, 121(2):181-190.

14. Kapusi E, Hensel G, Coronado MJ, Broeders S, Marthe C, Otto I, Kumlehn J: The elimination of a selectable marker gene in the doubled haploid progeny of co-transformed barley plants. Plant Mol Biol 2013, 81(1-2):149-160.

15. Ravi M, Chan SW: Haploid plants produced by centromere-mediated genome elimination. Nature 2010, 464(7288):615-618.

16. Geiger HH, Gordillo GA: Doubled haploids in hybrid maize breeding. Maydica 2009, 54:485-499.

17. Munyon IP, Hubstenberger JF, Phillips GC: Origin of plantlets and callus obtained from chile pepper anther cultures. In Vitro Cell Dev Biol 1989, 25:293-296

18. Jankowicz-Cieslak J, Huynh OA, Bado S, Matijevic M, Till BJ: Reversegenetics by TILLING expands through the plant kingdom. Emirates Journal of Food and Agriculture 2011, 23(4):290-300

19. Till BJ, Zerr T, Comai L, Henikoff S: A protocol for TILLING and Ecotilling in plants and animals. Nat Protoc 2006, 1(5):2465-2477.

20. Till BJ, Jankowicz-Cieslak J, Sagi L, Huynh OA, Utsushi H, Swennen R, Terauchi R, Mba C: Discovery of nucleotide polymorphisms in the Musa gene pool by Ecotilling. Theor App/ Genet 2010, 121(7):1381-1389.

21. Jankowicz-Cieslak J, Huynh OA, Brozynska M, Nakitandwe J, Till BJ: Induction, rapid fixation and retention of mutations in vegetatively propagated banana. Plant Biotechnol J 2012, 10(9):1056-1066.

22. Slade AJ, McGuire C, Loeffler D, Mullenberg J, Skinner W, Fazio G, Holm A, Brandt KM, Steine MN, Goodstal JF, et al: Development of high amylose wheat through TILLING. BMC Plant Biol 2012, 12:69.

23. Kurowska M, Daszkowska-Golec A, Gruszka D, Marzec M, Szurman M, Szarejko I, Maluszynski M: TILLING - a shortcut in functional genomics. J Appl Genet 2011, 52(4):371-390

24. Sato Y, Shirasawa K, Takahashi Y, Nishimura M, Nishio T: Mutant Selection from Progeny of Gamma-ray-irradiated Rice by DNA Heteroduplex Cleavage using Brassica Petiole Extract. Breed Sci 2006, 56(2):179-183.

25. Till BJ, Burtner C, Comai L, Henikoff S: Mismatch cleavage by single-strand specific nucleases. Nucleic Acids Res 2004, 32(8):2632-2641.

26. Garvin MR, Gharrett AJ: DEco-TILLING: an inexpensive method for single nucleotide polymorphism discovery that reduces ascertainment bias. Mol Ecol Notes 2007, 7(5):735-746.

27. Raghavan C, Naredo MEB, Wang HH, Atienza G, Liu B, Qiu FL, McNally KL, Leung $\mathrm{H}$ : Rapid method for detecting SNPs on agarose gels and its application in candidate gene mapping. Mol Breeding 2007, 19(2):87-101.

28. Comai L, Young K, Till BJ, Reynolds SH, Greene EA, Codomo CA, Enns LC, Johnson JE, Burtner C, Odden AR, et al: Efficient discovery of DNA polymorphisms in natural populations by Ecotilling. Plant J 2004, 37(5):778-786.

29. Dunwell JM, Wilkinson MJ, Nelson S, Wening S, Sitorus AC, Mienanti D, Alfiko Y, Croxford AE, Ford CS, Forster BP, et al: Production of haploids and doubled haploids in oil palm. BMC Plant Biol 2010, 10:218.

30. Murovec J, Stajner N, Jakse J, Javornik B: Microsatellite Marker for Homozygosity Testing of Putative Doubled Haploids and Characterization of Mimulus Species Derived by a Cross-genera Approach. J Am Soc Hortic Sci 2007, 132(5):659-663.

31. Nelson MN, Mason AS, Castello MC, Thomson L, Yan G, Cowling WA: Microspore culture preferentially selects unreduced (2n) gametes from an interspecific hybrid of Brassica napus L. x Brassica carinata Braun. Theor Appl Genet 2009, 119(3):497-505.

32. Chani E, Veilleux RE, Boluarte-Medina T: Improved androgenesis of interspecific potato and efficiency of SSR markers to identify homozygous regenerants. Plant Cell Tiss Org Cult 2000, 60:101-112.

33. Varshney RK, Marcel TC, Ramsay L, Russell J, Roder MS, Stein N, Waugh R, Langridge P, Niks RE, Graner A: A high density barley microsatellite consensus map with 775 SSR loci. Theor Appl Genet 2007, 114(6):1091-1103.

34. Szurman-Zubrzycka M: Identification of new alleles of genes potentially related to root system development in Hordeum vulgare and their functional analysis. PhD thesis. Katowice. Poland: University of Silesia; 2013.

35. Gottwald S, Bauer P, Komatsuda T, Lundqvist U, Stein N: TILLING in the two-rowed barley cultivar 'Barke' reveals preferred sites of functional diversity in the gene HvHox1. BMC Res Notes 2009, 2:258.

36. Mejlhede N, Kyjovska Z, Backes G, Burhenne K, Rasmussen SK, Jahoor A: EcoTILLING for the identification of allelic variation in the powdery mildew resistance genes mlo and Mla of barley. Plant Breed 2006, 125(5):461-467.

37. Till BJ, Zerr T, Bowers E, Greene EA, Comai L, Henikoff S: High-throughput discovery of rare human nucleotide polymorphisms by Ecotilling. Nucleic Acids Res 2006, 34(13):e99.

38. Bulgarelli D, Biselli C, Collins NC, Consonni G, Stanca AM, Schulze-Lefert P, Vale G: The CC-NB-LRR-type Rdg2a resistance gene confers immunity to the seed-borne barley leaf stripe pathogen in the absence of hypersensitive cell death. PLoS One 2010, 5:9.

39. Cooper JL, Till BJ, Laport RG, Darlow MC, Kleffner JM, Jamai A, El-Mellouki T, Liu S, Ritchie R, Nielsen N, et al: TILLING to detect induced mutations in soybean. BMC Plant Biol 2008, 8:9.

doi:10.1186/1746-4811-9-43

Cite this article as: Hofinger et al:: Validation of doubled haploid plants by enzymatic mismatch cleavage. Plant Methods 2013 9:43.

\section{Submit your next manuscript to BioMed Central and take full advantage of:}

- Convenient online submission

- Thorough peer review

- No space constraints or color figure charges

- Immediate publication on acceptance

- Inclusion in PubMed, CAS, Scopus and Google Scholar

- Research which is freely available for redistribution

Submit your manuscript at www.biomedcentral.com/submit
C Biomed Central 